\title{
Effect of superposition and masking between red blood cell autoantibodies and alloantibodies
}

\author{
Y. Yu and D.Q. Wang \\ Department of Blood Transfusion, Chinese PLA General Hospital, \\ Haidian District, Beijing, China \\ Corresponding author: D.Q. Wang \\ E-mail: deqingwangcn@163.com
}

Genet. Mol. Res. 13 (2): 4666-4672 (2014)

Received June 18, 2013

Accepted January 16, 2014

Published June 18, 2014

DOI http://dx.doi.org/10.4238/2014.June.18.9

\begin{abstract}
This study aimed to explore the law of superposition and masking between autoantibodies and alloantibodies, and to ensure the detection of alloantibodies and to improve the safety of warm autoimmune hemolytic anemia patients. Eight kinds of commercial IgG red blood cell antibody reagents were serially diluted, and 3 kinds of antibodies at dilutions showing a continuous gradual decline in agglutination strength with the corresponding antigen red blood cells were treated as the target antibodies. Anti-D and anti-M were treated as simulated autoantibodies, and anti-Fy ${ }^{\mathrm{a}}$ was treated as a simulated alloantibody. Four concentrations, 4+, 3+, 2+ and 1+, of autoantibodies and three concentrations, $3+, 2+$ and $1+$, of alloantibodies were combined, and 12 kinds of hybrid antibodies were detected and evaluated by the anti-human globulin micro-column gel assay. When the simulated strong autoantibody $(4+)$ was used, the alloantibodies $(3+, 2+, 1+)$ had no effect on the final agglutination strength; when the strength of agglutination produced by the simulated autoantibody was less than $4+$, and at the same time there were alloantibodies $(3+, 2+, 1+)$, the differences in agglutination strength with a panel of RBCs could be clearly observed. Strong autoantibodies (4+) can exert a masking effect, leading to alloantibodies being undetected; autoantibodies less than 4+,
\end{abstract}


will produce the superimposed effect with alloantibodies, resulting in differences in agglutination strength.

Key words: Autoantibody; Alloantibodies; Superposition effect; Masking effect; Warm autoimmune hemolytic anemia

\section{INTRODUCTION}

Warm autoimmune hemolytic anemia (WAIHA) is a common kind of disease that usually requires repeated blood transfusion. When the patient with WAIHA has a broadly reactive autoantibody, it is difficult to find a completely compatible donor blood (Leger and Garratty, 1999; Laine et al., 2000; Garratty and Petz, 1993, 2002; Petz, 2003). There are multiple reports in the literature demonstrating that patients who have warm autoantibodies in their serum have a higher rate of alloimmunization (e.g., 12 to 40\%, with a mean of 32\%) (Branch and Petz, 1999; Young et al., 2004; Maley et al., 2005; Ahrens et al., 2007). These alloantibodies in WAIHA patients can lead to hemolysis, but the clinical symptoms may just be aggravated anemia, and the role of alloantibodies has been ignored because of the patient's own existing hemolytic anemia caused by the autoantibodies. For these patients, to find and identify red cell alloantibodies and screen the corresponding antigen-negative donor red blood cells during the blood transfusion compatibility testing process is an important means to ensure the safety of blood transfusion.

How do we find and identify these alloantibodies? It is relatively easy to identify a single red blood cell antibody by selecting a suitable red blood cell panel. But if there is an autoantibody at the same time, the autoantibody may mask the presence of red cell alloantibodies, resulting in the alloantibodies being missed or interference with their specific identification, and ultimately not ensuring that the donor red blood cells do not contain the corresponding antigen. The aim of this study was to simulate the different strength combinations of alloantibodies and autoantibodies to explore the law of superimposition and masking between autoantibodies and alloantibodies and to provide a reference to take further steps in the identification of alloantibodies. This would improve the detection rate of alloantibodies in WAIHA patients and reduce the risk caused by undetected alloantibodies.

\section{MATERIAL AND METHODS}

\section{Screening of IgG antibodies}

Eight kinds of commercial IgG blood group antibodies were serially diluted with saline, and the red blood cells containing the corresponding antigen were selected to identify the activities of antibodies diluted. The agglutination strength of each blood group antibody at different dilutions with the corresponding antigen red blood cell was determined by using the anti-human globulin micro-column gel assay, where the antibodies that showed continuous gradual decline in agglutination were treated as the target antibodies.

\section{Evaluation on dose-effect of the target antibodies}

The aforementioned screening of blood group antibodies was carried out to evaluate 
the dose effect of the target antibodies. The agglutination strength produced by a panel of RBCs containing the corresponding homozygous and heterozygous antigen and diluted antibodies that showed $2+$ agglutination with the corresponding homozygous antigen red blood cells was determined using the anti-human globulin micro-gel method. The dose effect of three target antibodies was evaluated.

\section{Choice of simulated autoantibodies}

IgG anti-D (4+) and IgG anti-M (3+, 2+, 1+) antibodies were treated as simulated autoantibodies according to the result of aforementioned screening tests.

\section{Choice of alloantibodies}

IgG anti-Fy antibody $(3+, 2+, 1+)$ was treated as an alloantibody according to the result of aforementioned screening tests.

\section{Combinations of simulated autoantibodies and alloantibodies}

$\operatorname{IgG}$ anti-D antibody $(4+)$, and $\operatorname{IgG}$ anti-M $(3+, 2+, 1+)$ as simulated autoantibodies and $\operatorname{IgG}$ anti-Fy $(3+, 2+, 1+)$ as simulated alloantibody, were used to obtain 12 modes of combined antibodies (Table 1).

Table 1. Twelve combinations of autoantibodies and alloantibodies.
\begin{tabular}{lcccc}
\hline & IgG anti-D (1:32)4+ & IgG anti-M (1:4)3+ & IgG anti-M (1:8)2+ & IgG anti-M (1:16)1+ \\
\hline IgG anti-Fya $(1: 4) 3+$ & Group 1 & Group 2 & Group 3 & Group 4 \\
IgG anti-Fy $(1: 8) 2+$ & Group 5 & Group 6 & Group 7 & Group 8 \\
IgG anti-Fy $(1: 16) 1+$ & Group 9 & Group 10 & Group 11 & Group 12 \\
\hline
\end{tabular}

\section{RESULTS}

\section{Activities of 8 kinds of IgG blood group antibodies}

Only IgG anti-M and anti-Fy of eight kinds of blood group antibodies showed a continuous gradual decline in agglutination strength with fold dilution (Table 2).

\begin{tabular}{|c|c|c|c|c|c|c|c|c|c|c|c|c|}
\hline & $1: 1$ & $1: 2$ & $1: 4$ & $1: 8$ & $1: 16$ & $1: 32$ & $1: 64$ & $1: 128$ & $1: 256$ & $1: 512$ & $1: 1024$ & $1: 2048$ \\
\hline IgG anti-D & $4+$ & $4+$ & $4+$ & $4+$ & $4+$ & $4+$ & $3+$ & $3+$ & $3+$ & $3+$ & $1+$ & 0 \\
\hline IgG anti-k & $3+$ & $3+$ & $3+$ & $1+$ & $1+$ & $1+$ & \pm & 0 & - & - & - & - \\
\hline IgG anti-M & $4+$ & $3+$ & $3+$ & $2+$ & $1+$ & \pm & 0 & 0 & - & - & - & - \\
\hline IgG anti-N & $4+$ & $4+$ & $3+$ & $3+$ & $1+$ & $1+$ & \pm & 0 & - & - & - & - \\
\hline IgG anti-S & $3+$ & $3+$ & $3+$ & $3+$ & $2+$ & $2+$ & $2+$ & 0 & - & - & - & - \\
\hline IgG anti-Jk ${ }^{\mathrm{a}}$ & $3+$ & $3+$ & $3+$ & $3+$ & $1+$ & 0 & 0 & 0 & - & - & - & - \\
\hline IgG anti- Fy & $3+$ & $3+$ & $3+$ & $2+$ & $1+$ & 0 & 0 & 0 & - & - & - & - \\
\hline IgG anti- Fy & $4+$ & $3+$ & $2+$ & 0 & 0 & 0 & 0 & 0 & - & - & - & - \\
\hline
\end{tabular}




\section{Evaluation}

Two kinds of diluted antibodies showed $2+$ agglutination with red blood cells containing the corresponding homozygous and heterozygous antigens, respectively. There was no significant difference in strength between the two kinds of diluted antibodies.

\section{Simulated mixture of autoantibodies and alloantibodies}

When the simulated strong autoantibody $(4+)$ existed, the alloantibodies $(3+, 2+, 1+)$ had no effect on the final agglutination strength; when the strength of agglutination caused by the simulated autoantibody was less than $4+$, in the presence of alloantibodies $(3+, 2+, 1+)$, the differences in agglutination strength with a panel of RBCs could be clearly observed (Tables 3 and 4). When strong autoantibodies $(4+)$ were combined with alloantibodies $(3+, 2+$ and $1+)$, the differences in agglutination strength were evident after a certain degree of dilution. Alloantibodies could be found by this method, but we could not identify the specificities (Table 5).

Table 3. Agglutination strength of the combination of simulated strong autoantibodies $(4+)$ and alloantibodies $(3+, 2+, 1+)$.

\begin{tabular}{lccccccc}
\hline Group & $\mathrm{D}+/ \mathrm{Fy}^{\mathrm{a}}+$ & $\mathrm{D}+/ \mathrm{Fy}^{\mathrm{a}}-$ & $\mathrm{D}+/ \mathrm{Fy}^{\mathrm{a}}+$ & $\mathrm{D}+/ \mathrm{Fy}^{\mathrm{a}}-$ & $\mathrm{D}+/ \mathrm{Fy}^{\mathrm{a}}-$ & $\mathrm{D}+/ \mathrm{Fy}^{\mathrm{a}}+$ & $\mathrm{D}+/ \mathrm{Fy}^{\mathrm{a}}+$ \\
\hline 1 & $4+$ & $4+$ & $4+$ & $4+$ & $4+$ & $4+$ & $4+$ \\
5 & $4+$ & $4+$ & $4+$ & $4+$ & $4+$ & $4+$ & $4+$ \\
9 & $4+$ & $4+$ & $4+$ & $4+$ & $4+$ & $4+$ & $4+$ \\
\hline
\end{tabular}

\begin{tabular}{|c|c|c|c|c|c|c|c|c|c|c|}
\hline Group & $\mathrm{M}+/ \mathrm{Fy}^{\mathrm{a}}-$ & $\mathrm{M}+/ \mathrm{Fy}^{\mathrm{a}}+$ & $\mathrm{M}+/ \mathrm{Fy}^{\mathrm{a}}-$ & $\mathrm{M}+/ \mathrm{Fy}^{\mathrm{a}}+$ & $\mathrm{M}+/ \mathrm{Fy}^{\mathrm{a}}-$ & $\mathrm{M}+/ \mathrm{Fy}^{\mathrm{a}}-$ & $\mathrm{M}+/ \mathrm{Fy}^{\mathrm{a}}+$ & $\mathrm{M}+/ \mathrm{Fy}^{\mathrm{a}}-$ & $\mathrm{M}+/ \mathrm{Fy}^{\mathrm{a}}+$ & $\mathrm{M}+/ \mathrm{Fy}^{\mathrm{a}}+$ \\
\hline 2 & $3+$ & $4+$ & $3+$ & $4+$ & $3+$ & $3+$ & $4+$ & $3+$ & $4+$ & $4+$ \\
\hline 3 & $2+$ & $4+$ & $2+$ & $4+$ & $2+$ & $2+$ & $4+$ & $2+$ & $4+$ & $4+$ \\
\hline 4 & $1+$ & $3+$ & $1+$ & $3+$ & $1+$ & $1+$ & $3+$ & $1+$ & $3+$ & $3+$ \\
\hline 6 & $3+$ & $4+$ & $3+$ & $4+$ & $3+$ & $4+$ & $3+$ & $4+$ & $3+$ & $4+$ \\
\hline 7 & $2+$ & $3+$ & $2+$ & $3+$ & $2+$ & $2+$ & $3+$ & $2+$ & $3+$ & $3+$ \\
\hline 8 & $1+$ & $3+$ & $1+$ & $3+$ & $1+$ & $1+$ & $3+$ & $1+$ & $3+$ & $3+$ \\
\hline 10 & $3+$ & $4+$ & $3+$ & $4+$ & $3+$ & $3+$ & $4+$ & $3+$ & $4+$ & $4+$ \\
\hline 11 & $2+$ & $3+$ & $2+$ & $3+$ & $2+$ & $2+$ & $3+$ & $2+$ & $3+$ & $3+$ \\
\hline 12 & \pm & $2+$ & $1+$ & $2+$ & \pm & \pm & $2+$ & \pm & $2+$ & $2+$ \\
\hline
\end{tabular}

\begin{tabular}{|c|c|c|c|c|c|c|c|}
\hline Group (ratio) & $\mathrm{D}+/ \mathrm{Fy}^{\mathrm{a}}+$ & $\mathrm{D}+/ \mathrm{Fy}^{\mathrm{a}}-$ & $\mathrm{D}+/ \mathrm{Fy}^{\mathrm{a}}+$ & $\mathrm{D}+/ \mathrm{Fy}^{\mathrm{a}}-$ & $\mathrm{D}+/ \mathrm{Fy}^{\mathrm{a}}-$ & $\mathrm{D}+/ \mathrm{Fy}^{\mathrm{a}}+$ & $\mathrm{D}+/ \mathrm{Fy}^{\mathrm{a}}+$ \\
\hline $1(1: 2)$ & $4+$ & $3+$ & $4+$ & $3+$ & $3+$ & $4+$ & $4+$ \\
\hline $1(1: 4)$ & $4+$ & $3+$ & $4+$ & $3+$ & $3+$ & $4+$ & $4+$ \\
\hline $1(1: 8)$ & $3+$ & $3+$ & $3+$ & $3+$ & $3+$ & $3+$ & $3+$ \\
\hline $1(1: 16)$ & $3+$ & $3+$ & $3+$ & $3+$ & $3+$ & $3+$ & $3+$ \\
\hline $5(1: 2)$ & $4+$ & $3+$ & $4+$ & $3+$ & $3+$ & $4+$ & $4+$ \\
\hline $5(1: 4)$ & $3+$ & $3+$ & $3+$ & $3+$ & $3+$ & $3+$ & $3+$ \\
\hline $5(1: 8)$ & $3+$ & $3+$ & $3+$ & $3+$ & $3+$ & $3+$ & $3+$ \\
\hline $5(1: 16)$ & $3+$ & $3+$ & $3+$ & $3+$ & $3+$ & $3+$ & $3+$ \\
\hline $9(1: 2)$ & $4+$ & $3+$ & $4+$ & $3+$ & $3+$ & $4+$ & $4+$ \\
\hline $9(1: 4)$ & $3+$ & $3+$ & $3+$ & $3+$ & $3+$ & $3+$ & $3+$ \\
\hline $9(1: 8)$ & $3+$ & $3+$ & $3+$ & $3+$ & $3+$ & $3+$ & $3+$ \\
\hline $9(1: 16)$ & $3+$ & $3+$ & $3+$ & $3+$ & $3+$ & $3+$ & $3+$ \\
\hline
\end{tabular}




\section{DISCUSSION}

Patients with WAIHA generally produce autoantibodies that can make their hemoglobin level show a slow progressive decline because of destruction of their own red blood cells, eventually leading to severe anemia and even death. These autoantibodies can react with the patient's own red blood cells and all donors' red blood cells, and the activity of autoantibodies is basically consistent, where there is no specificity for these autoantibodies, which usually leads to the blood bank not finding any serological consistency of donor red blood cells. It is then difficult for blood transfusion officers and clinicians to make transfusion decisions. The usual practice is to wait even until the hemoglobin level is very low in patients with lifethreatening conditions before deciding on transfusion. In fact, the destruction of allogeneic red blood cells by autoantibodies is not stronger than their own red blood cells. In other words, the autoantibodies do not aggravate the patient's hemolytic anemia because of allogeneic transfusion, and allogeneic transfusion still benefits patients (Salama et al., 1992; Garratty and Petz, 1993; Petz and Garratty, 2004). However, there is a serological characteristic in patients with WAIHA that needs attention. The frequency of alloantibodies in patients with WAIHA was significantly higher than that in patients without WAIHA. The existence of alloantibodies increases the risk of blood transfusion in patients with WAIHA, and autoantibodies may mask alloantibodies, which results in alloantibodies being missed and the occurrence of hemolytic reaction (Jenner and Holland, 1996; Petz, 1996; Church et al., 2000; Drouillard, 2008).

For patients with autoantibodies and alloantibodies, it is even more important to detect alloantibodies. Generally, autoantibodies are absorbed and removed by using patient's own red blood cells, which reveal the specificity of alloantibodies (Branch and Petz, 1999; Leger and Garratty, 1999; Garratty and Petz, 2002; Drouillard, 2008) and the corresponding antigennegative red blood cells are transfused to improve the safety and efficacy of blood transfusion. In fact, for the majority of WAIHA patients, it is very difficult to collect sufficient blood samples for the absorption of autoantibodies because of their own severe anemia (Issitt et al., 1996). Therefore, it is unrealistic to identify alloantibodies for each WAIHA patient by the self-absorbing technique. Past experience told us that, if the reactivity of alloantibodies was higher than that of autoantibodies, the interference of autoantibodies can be gradually reduced until it disappears by the method of continuous dilution, so that the specificity of the alloantibodies can be shown (Buetens and Ness, 2003; Petz, 2004; Leger, 2008). However, in theory, if the activity of autoantibodies is higher than or close to that of autoantibodies, this may lead to alloantibodies being masked and undetected. If the dilution is carried out, and the activity of alloantibodies and autoantibodies will simultaneously decrease, the activity of the alloantibodies will disappear first, making it impossible to detect and identify them (Laine et al., 2000).

In this study, the serological characteristics of mixed antibodies under different reactivity conditions (agglutination strength) were determined, and the law of the effect of superposition and masking of alloantibodies and autoantibody was revealed to prevent the alloantibodies from being missed. We evaluated eight kinds of commercially available IgG blood group antibodies (see Table 2), and found that IgG anti-M and IgG anti-Fy a showed a continuous gradual decline in agglutination with dilution. However, the activity of $\mathrm{IgG}$ anti-M antibody was too low, where only the original solution could make the corresponding red blood cells show 4+ agglutination, and could not be mixed with other antibodies. $\operatorname{IgG}$ anti-D $(4+, 1: 32)$ and $\operatorname{IgG}$ anti-M $(3+, 2+, 1+)$ were together treated as simulated autoantibody $(4+, 3+, 2+, 1+)$, and $\operatorname{IgG}$ 
anti-Fy antibody $(3+, 2+, 1+)$ was treated as simulated alloantibodies to obtain 12 combinations of autoantibody and alloantibodies (Table 1). According to the features of autoantibody that can react with all donors' red blood cells to give the same agglutination strength, an appropriate panel of RBCs was selected to detect the agglutination strength of the 12 combinations of antibodies. Table 3 showed that strong autoantibodies (4+) could make all cells show 4+ agglutination, and that the specificity of alloantibodies was masked and missed. In this case, the superposition effect of alloantibodies and autoantibodies could be exposed by the continuous dilution method, and then the difference in agglutination strength was found and the presence of alloantibodies was confirmed (Table 5), but this method still could not directly identify the specificity of alloantibodies. Table 4 showed that if the reactivity of the autoantibody were less than $4+$, regardless of the activity of alloantibodies (1+ to 3+), the superposition effect between the antibodies would be shown, and a significant difference in agglutination strength would be detected to confirm the presence of alloantibodies. However, the identification of the specificity of alloantibodies still needs to exclude the interference of autoantibodies by the self-absorbing method. If sufficient red blood cells for the self-absorbing test cannot be obtained, the test will not be carried out. To confirm the safety of blood transfusion, another method can also be tried. Blood group antigen phenotypes of the recipient and donors should be fully identified to find the same or compatible red blood cell donor, which can circumvent the risk of alloantibodies (Garratty and Petz, 2002; Shirey et al., 2002). Of course, it may be very difficult to obtain a variety of rare serum reagents for most blood bank laboratories, but the blood supply services should have the ability to identify the blood group phenotype.

In summary, during the identification of antibody specificity in patients with WAIHA, if the agglutination strength of a panel of RBCs appeared inconsistent, there was the possibility of autoantibody in combination with alloantibodies; if there is the same agglutination strength for a panel of RBCs, and at the same time the agglutination strength is less than 4+, the merger presence of alloantibodies is very unlikely, even in the presence of alloantibodies, their activity will be low, and may not have clinical significance; if the agglutination strength for a panel of RBCs appears consistently 4+, the possibility of the existence of alloantibodies cannot be ruled out. In response to this case, the consecutive dilution of the serum or plasma of the patients should be performed. The lack of difference in activity for the diluted serum would prove the absence of alloantibodies; differences in agglutination strength would confirm the presence of alloantibodies, calling for further antibody identification tests.

\section{REFERENCES}

Ahrens N, Pruss A, Kähne A, Kiesewetter H, et al. (2007). Coexistence of autoantibodies and alloantibodies to red blood cells due to blood transfusion. Transfusion 47: 813-816.

Branch DR and Petz LD (1999). Detecting alloantibodies in patients with autoantibodies. Transfusion 39: 6-10.

Buetens OW and Ness PM (2003). Red blood cell transfusion in autoimmune hemolytic anemia. Curr. Opin. Hematol. 10: 429-433.

Church AT, Nance SJ and Kavitsky DM (2000). Predicting the presence of new alloantibodies underlying a warm autoantibody (abstract). Transfusion 40: 121S.

Drouillard DD (2008). Transfusion therapy for autoimmune hemolytic anemia patients: a laboratory perspective. Clin. Lab. Sci. 21: 7-11.

Garratty G and Petz LD (1993). Transfusing patients with autoimmune haemolytic anaemia. Lancet 341: 1220.

Garratty G and Petz LD (2002). Approaches to selecting blood for transfusion to patients with autoimmune hemolytic anemia. Transfusion 42: 1390-1392.

Issitt PD, Combs MR, Bumgarner DJ, Allen J, et al. (1996). Studies of antibodies in the sera of patients who have made 
red cell autoantibodies. Transfusion 36: 481-486.

Jenner PW and Holland PV (1996). Diagnosis and Management of Transfusion Reactions. In: Clinical Practice of Transfusion Medicine. (Petz LD, Swisher SN, Kleinman S, Spence RK, et al., eds.). Churchill-Livingstone, New York, 905-929.

Laine EP, Leger RM, Arndt PA, Calhoun L, et al. (2000). In vitro studies of the impact of transfusion on the detection of alloantibodies after autoadsorption. Transfusion 40: 1384-1387.

Leger RM (2008). The Positive Direct Antiglobulin Test and Immune-Mediated Hemolysis. In: 521 AABB Technical Manual. 16th edn, Bethesda, -499 .

Leger RM and Garratty G (1999). Evaluation of methods for detecting alloantibodies underlying warm autoantibodies. Transfusion 39: 11-16.

Maley M, Bruce DG, Babb RG, Wells AW, et al. (2005). The incidence of red cell alloantibodies underlying panreactive warm autoantibodies. Immunohematology 21: 122-125.

Petz LD (1996). Blood Transfusion in Acquired Hemolytic Anemias. In: Clinical Practice of Transfusion Medicine. (Petz LD, Swisher SN, Kleinman S, Spence RK, et al., eds.). Churchill Livingstone, New York: 469-499.

Petz LD and Garratty G (2004). Blood Transfusion in Autoimmune Hemolytic Anemias. In: Immune Hemolytic Anemias (Petz LD, Swisher SN, Kleinman S, Spence RK, et al., eds.). Churchill Livingstone, New York, 375-400.

Petz LD (2003). "Least incompatible" units for transfusion in autoimmune hemolytic anemia: should we eliminate this meaningless term? A commentary for clinicians and transfusion medicine professionals. Transfusion 43: 1503-1507.

Petz LD (2004). A physician's guide to transfusion in autoimmune haemolytic anaemia. Br. J. Haematol. 124: 712-716.

Salama A, Berghofer H and Mueller-Eckhardt C (1992). Red blood cell transfusion in warm-type autoimmune haemolytic anaemia. Lancet 340: 1515-1517.

Shirey RS, Boyd JS, Parwani AV, Tanz WS, et al. (2002). Prophylactic antigen-matched donor blood for patients with warm autoantibodies: an algorithm for transfusion management. Transfusion 42: 1435-1441.

Young PP, Uzieblo A, Trulock E, Lublin DM, et al. (2004). Autoantibody formation after alloimmunization: are blood transfusions a risk factor for autoimmune hemolytic anemia? Transfusion 44: 67-72. 